\title{
Neuroprotective effects and intracellular signaling pathways of erythropoietin in a rat model of multiple sclerosis
}

\author{
MB Sättler (née Hobom) ${ }^{\star, 1}$, D Merkler ${ }^{2}$, K Maier ${ }^{1}$, C Stadelmann ${ }^{2}$, \\ H Ehrenreich ${ }^{3}$, M Bähr ${ }^{1}$ and R Diem ${ }^{1}$ \\ ${ }^{1}$ Neurologische Universitätsklinik, Robert-Koch-Str. 40, 37075 Göttingen, \\ Germany \\ ${ }^{2}$ Institut für Neuropathologie, Universitätsklinik, Robert-Koch-Str. 40, 37075 \\ Göttingen, Germany \\ ${ }^{3}$ Max-Planck-Institut für Experimentelle Medizin, Hermann-Rein-Str. 3, 37075 \\ Göttingen, Germany \\ * Corresponding author: M Sättler, Neurologische Universitätsklinik, Robert- \\ Koch-Str. 40, 37075 Göttingen, Germany. Tel: + 4955139 6603; \\ Fax: + 4955139 8405; E-mail: msaett|@gwdg.de
}

Received 16.3.04; revised 19.7.04; accepted 29.7.04; published online 1.10.04 Edited by $\mathrm{G}$ Ciliberto

\begin{abstract}
In multiple sclerosis (MS), long-term disability is primarily caused by axonal and neuronal damage. We demonstrated in a previous study that neuronal apoptosis occurs early during experimental autoimmune encephalomyelitis, a common animal model of MS. In the present study, we show that, in rats suffering from myelin oligodendrocyte glycoprotein (MOG)-induced optic neuritis, systemic application of erythropoietin (Epo) significantly increased survival and function of retinal ganglion cells (RGCs), the neurons that form the axons of the optic nerve. We identified three independent intracellular signaling pathways involved in Epo-induced neuroprotection in vivo: Protein levels of phospho-Akt, phospho-MAPK 1 and 2, and $\mathrm{Bcl}-2$ were increased under Epo application. Using a combined treatment of Epo together with a selective inhibitor of phosphatidylinositol 3-kinase ( $\mathrm{PI3}-\mathrm{K}$ ) prevented upregulation of phospho-Akt and consecutive RGC rescue. We conclude that in MOG-EAE the PI3-K/Akt pathway has an important influence on RGC survival under systemic treatment with Epo. Cell Death and Differentiation (2004) 11, S181-S192.

doi:10.1038/sj.cdd.4401504

Published online 1 October 2004
\end{abstract}

Keywords: optic neuritis; erythropoietin; retinal ganglion cells; Bcl-2 family; phosphatidylinositol 3-kinase

Abbreviations: BBB, blood brain barrier; BN, brown Norway; CFA, complete Freund's adjuvant; EAE, experimental autoimmune encephalomyelitis; Epo, erythropoietin; EpoR, erythropoietin receptor; ERG, electroretinogram; FG, Fluorogold; i.p., intraperitoneal; MAPK, mitogen-activated protein kinase; MBP, myelin basic protein; MEK, mitogen-activated protein kinase kinase; MOG, myelin oligodendrocyte glycoprotein; MS, multiple sclerosis; ON, optic nerve; PI-3K, phosphatidylinositol 3-kinase; RGCs, retinal ganglion cells; VEP, visual evoked potential; WM, wortmannin

\section{Introduction}

Multiple sclerosis (MS) is an autoimmune disease of the CNS that has long been thought to be primarily characterized by inflammation and demyelination. In addition, irreversible axonal damage was observed in postmortem and MRI studies at different stages of the disease $e^{1-4}$ and is assumed to be an essential cause of nonremitting clinical disability and disease progression. ${ }^{5}$ In addition to severe axonal pathology, apoptotic neuronal cell death occurs in demyelinated cerebral cortex lesions of MS patients. ${ }^{6}$ So far, no therapy has been established to prevent axonal and neuronal injury in this neuro-inflammatory disease.

Experimental autoimmune encephalomyelitis (EAE) induced by myelin oligodendrocyte glycoprotein (MOG) is an established animal model of MS closely representing the pathogenetic lesion formation in this disease.$^{7-9} \operatorname{In}$ a previous study, we demonstrated that in this animal model neuritis of the optic nerve $(\mathrm{ON})$ provokes apoptotic retinal ganglion cell (RGC) death and thereby leads to a severe impairment of visual functions. ${ }^{10}$ Recently, we showed that high-dose methylprednisolone treatment, the standard therapy of acute MS relapses, increases neurodegeneration in MOG-EAE by inhibiting an endogenous neuroprotective pathway in RGCs. ${ }^{11}$ In this model, axonal damage has been shown to be qualitatively and quantitatively similar to the one observed in MS patients. ${ }^{12}$ Therefore, MOG-EAE seems to be an appropriate animal model for testing neuroprotective therapies that might prevent the development of progressive disability.

Erythropoietin (Epo) is the main regulator of erythropoiesis in mammals. ${ }^{13}$ It has been shown to reduce apoptosis of erythroid progenitors by interacting with a high-affinity receptor on these cells. ${ }^{14}$ Several intracellular signal transduction cascades are involved in the antiapoptotic effects of Epo on erythroblasts including Ras-mitogen-activated protein kinase (MAPK), phosphatidylinositol 3-kinase (PI3-K), and the Bcl-2 family. ${ }^{14,15}$ It is becoming increasingly clear that Epo has additional neuroprotective effects in different in vivo models of brain injury, ${ }^{16-19}$ modulating the same signal transduction cascades that we identified to be involved in RGC death in our MOG-EAE model, the Bcl-2 family and the PI3-K/Akt pathway. ${ }^{10}$ Furthermore, Epo has been demonstrated to delay the onset and to reduce the severity of clinical symptoms in an EAE model induced by myelin basic protein (MBP). ${ }^{19}$ In a recent study, it has been shown that these effects were caused by an Epo-induced modulation of cytokine expression. ${ }^{20}$ But, the potential effects of Epo on axonal damage and neuronal survival in EAE have not been investigated so far.

In the present study, we show for the first time in an animal model of MS that Epo is an effective neuroprotective therapy that promotes survival and function of RGCs, the neurons that form the axons of the ON. Moreover, we analyzed in detail the 
intracellular signal transduction cascades involved in Epodependent neuroprotection in vivo.

\section{Results}

\section{Effects of Epo treatment on the clinical severity of MOG-EAE}

Comparing the neurological deficits of rats with MOG-EAE under daily intraperitoneal (i.p.) injections of Epo $(5000 \mathrm{U} / \mathrm{kg})$ with those of vehicle-treated animals revealed no significant difference. With respect to our previous results which showed a severe degeneration of RGCs that started already before clinical onset of the disease, ${ }^{10}$ treatment was given from the day of immunization onward to prevent neuronal degeneration before it results in a functional deficit. Manifestation of the disease was slightly delayed in Epo-treated animals compared with the control group (day $15.7 \pm 0.8$ after immunization versus day $14.6 \pm 0.8$; mean \pm s.e.m.; $n=8$ for each group; $P=0.39$ ). During the first 3 days after clinical onset of $E A E$, the disease course was similar in both groups (data not shown). From day 5 after disease manifestation onward, there was a tendency to a reduced severity of neurological symptoms in Epo-treated animals, which resulted in a mean clinical score of $0.25 \pm 0.17$ in the Epo-treated group and $1.0 \pm 0.32$ (mean \pm s.e.m.; $n=6$ for each group; $P=0.07$ ) in the vehicle-treated animals at day 8 of EAE. The difference in the clinical outcome under daily application of Epo did not achieve statistical significance in MOG-EAE.

\section{RGC function determined by ERG recordings is improved in Epo-treated animals}

To examine the potential effects of Epo treatment on visual functions of rats during development of optic neuritis, we performed recordings of electroretinograms (ERGs) and visual evoked potentials (VEPs). Flash ERGs represent the electrophysiological answer of all retinal cells. In contrast, pattern ERGs evoked by stimulation with alternating black and white bars specifically correspond to the function of RGCs. ${ }^{21}$ We analyzed ERG potentials in response to pattern and flash stimuli at day 7 after immunization and at the day of clinical manifestation of the disease. Both eyes were measured separately in each animal. At day 7 after immunization, the mean of alternating bars in which a specific ERG potential was recordable was in the range of 3-6 bars in the vehicletreated group. This represents a visual acuity of $0.21 \pm 0.08$ cycles per degree (mean \pm s.e.m.; $n=8$; Figure 1a). In contrast, Epo-treated animals $(5000 \mathrm{U} / \mathrm{kg})$ showed retinal potentials in response to a mean of 12-24 discernable bars corresponding to visual acuity values of $0.52 \pm 0.18$ cycles per degree $(n=8)$. Moreover, RGC function in the Epo-treated group remained stable until manifestation of the disease. At day 1 of MOG-EAE, RGC function in the control group had further declined to a visual acuity of $0.10 \pm 0.07$ cycles per degree, whereas the Epo-treated rats produced pattern ERGs representing a significantly better visual acuity of $0.53 \pm 0.16$ cycles per degree $(P<0.05$; Figure $1 \mathrm{a})$. In this group, only one out of eight measured eyes had lost its ability to respond to pattern stimulation at day 1 of MOG-EAE, a

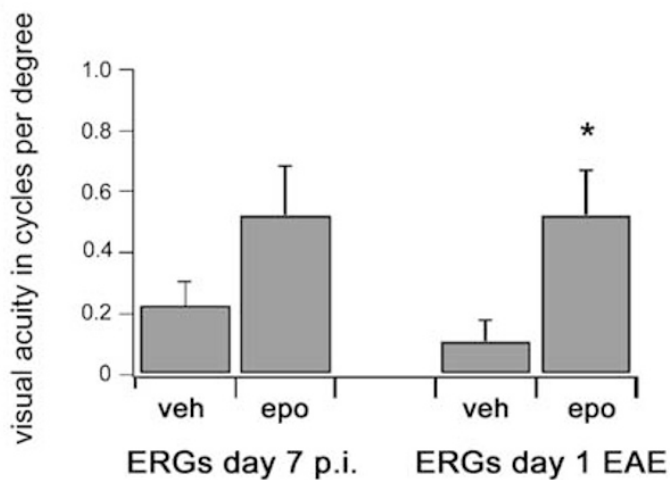

b



C

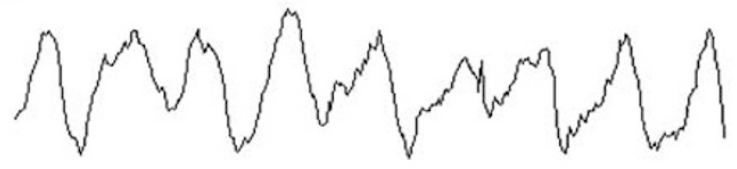

d

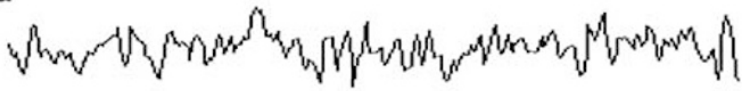

$20 \mu \mathrm{V}$

\section{$100 \mathrm{~ms}$}

Figure 1 ERG and VEP recordings following daily treatment with Epo or vehicle. (a) Pattern ERGs at day 7 after immunization (day 7 p.i.) and day 1 of MOG-EAE (day 1 EAE) in Epo (epo) and vehicle (veh)-treated animals. The $y$ axis shows the visual acuity values, data are given as the mean \pm s.e.m. *Statistically significant when compared with vehicle-treated controls (Student's $t$ test; $P<0.05)$. (b) Visual acuities of VEPs evoked by pattern stimulation at day 7 after immunization and at day 1 of MOG-EAE in vehicle and Epo-treated animals. (c) ERG of an Epo-treated rat at day 1 of MOG-EAE evoked by stimulation with 12 alternating bars. (d) ERG of a vehicle-treated animal at day 1 of MOG-EAE evoked by stimulation with 12 alternating bars. Only background noise levels of electrical activity were detectable

whereas in the vehicle-treated animals only two out of eight eyes still showed detectable ERG potentials in response to pattern stimulation. An ERG response to flash stimulation was 
detectable in all animals, indicating an intact function of the whole retina.

In order to investigate the effects of Epo treatment on the function of the ON itself, we simultaneously performed VEP recordings in both animal groups. With VEP flash stimulation axonal signaling of the $\mathrm{ON}$ was tested, indicating the animal's ability to discriminate between light and dark. At both time points, all control and Epo-treated animals showed cortical potentials in response to flash stimulation. Similar to the ERG experiments, pattern VEPs were recorded to estimate the animal's visual acuity. We have recently shown that healthy sham-immunized rats have visual acuity values of $1.31 \pm 0.16$ cycles per degree. ${ }^{22}$ At the clinical onset of MOG-EAE, we observed a severe decline of specific cortical potentials in response to pattern stimulation in this previous study. In our present study, at day 7 after immunization the mean of discernable alternating bars with detectable VEP responses was located within the range of $6-12$ bars $(n=8$ for each group) in the vehicle-treated as well as in the Epo-treated group. This corresponds to visual acuity values of $0.42 \pm 0.11$ versus $0.43 \pm 0.17$ cycles per degree (mean \pm s.e.m.; Figure 1b). The consistency of the VEP results in the different treatment groups did not change until day 1 of MOG-EAE. At this time point, both animal groups showed detectable cortical potentials evoked by pattern stimulation with a mean of 3-6 discernable bars (visual acuity of $0.33 \pm 0.09$ cycles per degree in the Epo-treated group versus $0.27 \pm 0.10$ cycles per degree in the vehicle-treated controls; Figure 1b). These VEP results indicate a severe impairment of $\mathrm{ON}$ function in both animal groups. In contrast to the ERG results which showed an improvement under Epo application, Epo did not improve the VEPs at both time points analyzed, nor did it stop the continuing loss of detectable VEPs between day 7 after immunization and day 1 of MOG-EAE.

\section{Histopathology of the ONs is unaffected by applications of Epo}

Investigating the severity of demyelination and inflammatory infiltration within the ONs at day 8 of MOG-EAE, we detected no noteworthy differences in both treatment groups. In Epotreated rats $(5000 \mathrm{U} / \mathrm{kg})$, the mean percentage of the demyelinated area determined by Luxol-fast blue staining with respect to the whole transverse cross section of the ON was $70.4 \pm 17.4$ (mean \pm s.e.m.; $n=6$ ). In the vehicle-treated group, we observed $85.8 \pm 12.5 \%(n=6)$ of demyelination in vertical ON sections. Additionally, the mean numbers of ON axons per square millimeter were in the same range in Epotreated animals compared with vehicle-treated controls $(6337 \pm 876$ versus $6546 \pm 593$; mean \pm s.e.m.; $n=6$ for each group). These results are in accordance with our VEP recordings, demonstrating no benefit in $\mathrm{ON}$ function under daily injections of Epo.

\section{Epo treatment increases RGC survival in MOG- EAE}

Based on our electrophysiological results which showed a functional improvement of RGCs under Epo treatment, we now investigated the potential effects of daily applications of 5000 U Epo per kilogram bodyweight on RGC survival. We compared the number of RGCs retrogradely labeled with Fluorogold (FG) in rats treated with Epo with those of vehicletreated animals at days 1 and 8 of MOG-EAE. In healthy Brown Norway (BN) rats immunized with complete Freund's adjuvant (CFA), $2730 \pm 145$ RGCs per square millimeter were detectable (mean \pm s.e.m.; $n=8$; Figure $2 a$ ). ${ }^{22}$ In the vehicletreated EAE group, we observed $1262 \pm 74$ RGCs per square millimeter $(n=8)$ at day 1 of MOG-EAE, corresponding to a survival of $46 \%$ of RGCs when compared to healthy controls. In contrast, RGC counts were significantly increased to $1917 \pm 99$ RGCs per square millimeter $(n=8 ; P<0.05$; Figure $2 a$ and $b$ ) at day 1 of the disease in the Epo-treated group of rats. This represents a survival rate of $70 \%$ of RGCs. The neuroprotective effect of Epo in MOG-EAE was reproduced in our second animal group that was followed until day 8 of MOGEAE. At this time point, in vehicle-treated rats the survival rate of RGCs had further dropped $(829 \pm 59$ RGCs per square millimeter; $n=8$; Figure 2 a) to $30 \%$ of the RGC density of healthy controls. In the Epo-treated group, we observed significantly higher RGC counts of $1506 \pm 39$ RGCs per square millimeter $(n=8 ; P<0.05$; Figure $2 \mathrm{a})$, indicating that $55 \%$ of RGCs survived. These results point out that, in both animal groups that had received Epo applications, a rescue of about $50 \%$ of RGCs that underwent neuronal cell death in the corresponding vehicle-treated controls was observed.

In order to determine whether the neuroprotective effects of Epo in MOG-EAE are dose-responsive, we additionally analyzed RGC densities following daily intraperitoneal applications of 2000 and $10000 \mathrm{U}$ Epo per kilogram bodyweight. At day 1 of MOG-EAE, RGC counts in the group of rats that had received $2000 \mathrm{U}$ Epo per kilogram showed no significant difference from vehicle-treated controls $(1350 \pm 51$ RGCs per square millimeter; $n=8$; Figure $2 \mathrm{c}$ ). On the contrary, daily applications of $10000 \mathrm{U}$ Epo per kilogram significantly increased RGC counts to $1777 \pm 73$ RGCs per square millimeter $(n=8$; Figure 2c; $P<0.05)$ compared with the control group, but failed to significantly augment $R G C$ rescue when compared to the dosage of $5000 \mathrm{U}$ Epo. These data indicate that a daily dosage of at least $5000 \mathrm{U}$ Epo per kilogram bodyweight is necessary to achieve a significant neuroprotective effect in our MOG-EAE model. A further increase of the dosage does not augment this effect.

\section{Epo application reduces the number of TUNEL and active caspase-3-positive RGCs}

In addition to the above given densities of FG-positive RGCs, we performed TUNEL and caspase-3 staining of retinal sections comparable in size and location to quantify RGC apoptosis under Epo $(5000 \mathrm{U} / \mathrm{kg})$ or vehicle treatment at day 1 of MOG-EAE. RGCs were identified by retrograde $F G$ labeling. In the Epo-treated animals, 0.88 \pm 0.3 (mean \pm s.e.m.; $n=8$ ) TUNEL-positive RGCs per retinal slice were detectable (Figure 2d). In the control group, we found a significantly higher rate of RGCs with DNA fragmentation. In this group, 6.1 \pm 2.8 TUNEL-positive RGCs per slice were identified $(n=8 ; P<0.05)$. Immunostaining of activated 
caspase-3 revealed $4.1 \pm 0.6$ (mean \pm s.e.m.; $n=8$ ) active caspase-3-positive RGCs per section in the Epo-treated group, whereas $8.0 \pm 0.8$ active caspase-3-positive RGCs were detected in the corresponding control sections $(n=8$; Figure $2 d$ ). In retinal sections of healthy rats, no TUNEL or active caspase-3-positive RGCs were observed. The reduction of TUNEL-reactive and active caspase-3-positive RGCs demonstrates the antiapoptotic activity of Epo in MOG-EAE.

\section{Detection of Epo and its receptor in MOG-EAE}

To determine the physiological role of endogenous Epo in our animal model, we performed ELISAs of retinal homogenates obtained at day 15 after immunization. In pooled retinal

a

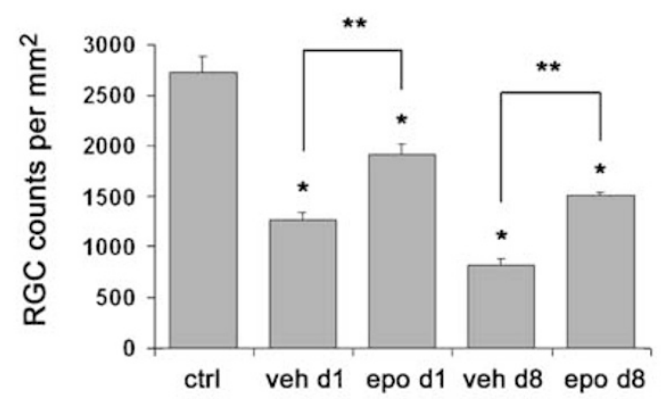

b
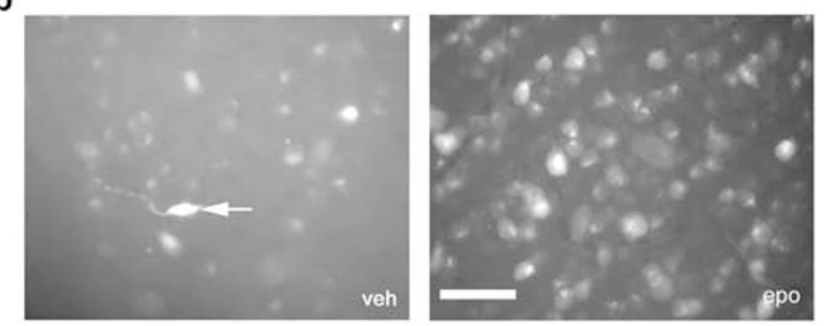

C

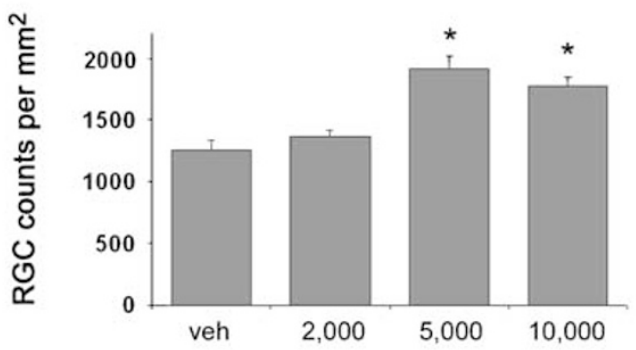

d

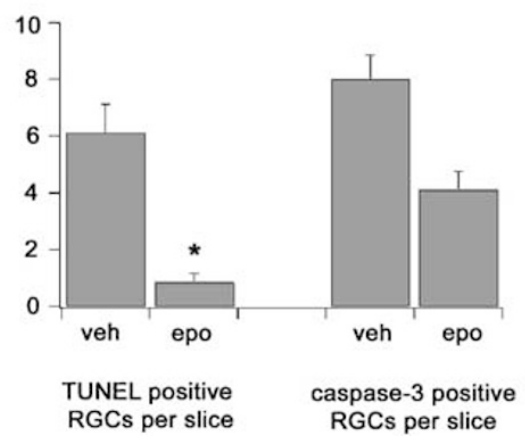

samples containing the retinas of six vehicle-treated rats each, we detected $41.4 \pm 2.2 \mathrm{mIU}$ Epo per milligram retinal tissue (mean \pm s.e.m.), indicating that in MOG-EAE a substantial endogenous production of Epo occurs within the retina. In comparison, in a group of Epo-treated rats (5000 U/ $\mathrm{kg} ; n=6)$, we observed $93.8 \pm 6.5 \mathrm{mlU}$ Epo per milligram retinal tissue $1 \mathrm{~h}$ after the last intraperitoneal application of the cytokine (mean \pm s.e.m.). These experiments revealed that Epo could reach its apparent target in our animal model, the RGCs, and was not stopped by the blood brain barrier (BBB).

In order to analyze whether Epo acts directly on RGCs or exerts its neuroprotective effects indirectly, for example, via influencing non-neuronal cell types during MOG-EAE, we investigated the expression of the Epo-receptor (EpoR) in the retina by immunohistochemistry (Figure $4 a$ ). In healthy control sections, no specific staining for the EpoR was detectable on RGCs. In contrast, at day 1 of MOG-EAE the majority of RGCs showed specific EpoR expression (Figure 4a). Epo applications $(5000 \mathrm{U} / \mathrm{kg})$ did not lead to a downregulation of the receptor, as shown by immunostaining of retinal sections at day 1 of MOG-EAE under Epo treatment.

\section{Epo activity on red blood cell counts}

As an in vivo assay for the specific activity of Epo, we analyzed the hematocrit levels at day 8 of MOG-EAE following Epo or vehicle treatment and compared them with healthy controls. Healthy rats showed hematocrit values within the normal range $(41.7 \pm 0.4 \%$; mean \pm s.e.m.; $n=5)$. On the contrary, daily intraperitoneal administration of Epo significantly increased the mean hematocrit to $65.0 \pm 3.8 \%(n=4 ; P<0.05)$, whereas in vehicle-treated rats at day 8 of MOG-EAE the mean hematocrit did not differ considerably from healthy animals $(42.8 \pm 1.0 \% ; n=4)$.

\section{Epo modulates three distinct intracellular neuroprotective pathways}

Based on our above given results which show a reduced number of apoptotic RGCs in animals treated with daily

Figure 2 Epo treatment significantly increased RGC density and reduced apoptosis of RGCs during MOG-EAE. (a) Data are given as the mean \pm s.e.m. of retrogradely labeled RGCs per square millimeter ( $y$-axis). RGC counts of vehicle (veh) and Epo-treated animals (epo) are indicated for days 1 (d 1) and 8 (d 8) of MOG-EAE and are compared with healthy controls (ctrl). *Statistically significant when compared with healthy controls; ${ }^{*}$ Statistically significant when compared with vehicle-treated animals (one-way ANOVA followed by Duncan's test; $P<0.05)$. Further significance was obtained comparing days 1 and 8 of vehicletreated rats as well as days 1 and 8 of Epo-treated animals $(P<0.05)$. (b) Representative retinal flat-mounts at 3/6 retinal radius from an Epo-treated animal (epo) and a vehicle-treated control (veh) at day 1 of MOG-EAE. RGCs were retrogradely labeled with Fluorogold. Note the occurrence of cells with ramificated dendritic processes corresponding to microglia (arrow) in the vehicletreated group. Scale bar: $40 \mu \mathrm{m}$. (c) Retrogradely labeled RGCs at day 1 of MOG-EAE (mean \pm s.e.m.). Comparison of vehicle treatment with the application of 2000,5000, or 10000 U Epo per kilogram bodyweight. *Statistically significant when compared with vehicle treatment or application of 2000 units Epo per kilogram (one-way ANOVA followed by Duncan's test; $P<0.05$ ). (d) Semiquantitative analysis of TUNEL positive (left bars) or active caspase-3positive (right bars) RGCs per retinal section at day 1 of MOG-EAE following Epo or vehicle treatment. *Statistically significant when compared with vehicle-treated animals (Student's $t$-test; $P<0.05$ ) 
injections of Epo $(5000 \mathrm{U} / \mathrm{kg})$, we now analyzed the involved intracellular signal transduction cascades in our MOG-EAE model. We examined the proteins phospho-Akt (p-Akt), phospho-MAPK (p-MAPK) 1 and 2, as well as two members of the Bcl-2 family, the antiapoptotic protein Bcl-2 and the proapoptotic member Bax. All these three signal transduction cascades have been shown to be involved in antiapoptotic effects of Epo on erythroblasts. ${ }^{14,15}$ Akt, a serine/threonine protein kinase, was identified as a downstream component in survival signaling through $\mathrm{PI}-\mathrm{K} .{ }^{23}$ Western blot analysis of retinal lysates of Epo-treated rats revealed a significant upregulation of p-Akt when compared to vehicle-treated controls (Figure 3a). The expression of the unphosphorylated, inactive Akt protein was the same in all samples analyzed.

MAPKs represent another family of serine/threonine protein kinases. These kinases build the end of an intracellular pathway involving multiple serine/threonine kinases that are activated in a cascade, including mitogen-activated kinase kinase (MEK). ${ }^{24}$ The phosphorylated forms of MAPK 1 and 2 were both increased in retinal protein lysates of Epo-treated animals compared with vehicle-treated controls (Figure $3 b$ ). Again, the expression of the unphosphorylated proteins was unaffected under Epo application.

Additionally, we analyzed the influence of Epo treatment on two members of the Bcl-2 family. The Bcl-2 family is a wellcharacterized group of apoptosis regulatory proteins that function in protein-protein interactions. ${ }^{25}$ By forming heterodimers, high amounts of Bax can antagonize the antiapoptotic activity of $\mathrm{Bcl}-2$ such that the ratio of $\mathrm{Bcl}-2$ to $\mathrm{Bax}$ determines survival or death following an apoptotic stimulus. ${ }^{26} \mathrm{We}$ observed that $\mathrm{Bcl}-2$ was strongly upregulated in protein lysates of retinas following Epo treatment (Figure 3c). In contrast, immunohistochemistry of retinal sections revealed that the amount of Bax protein in RGCs was reduced in Epotreated animals when compared with the corresponding controls (Figure 4b). Epo treatment of MOG-EAE modified the expression of these two proteins of the Bcl-2 family towards the antiapoptotic side. a pAkt
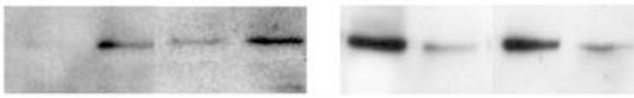

Akt
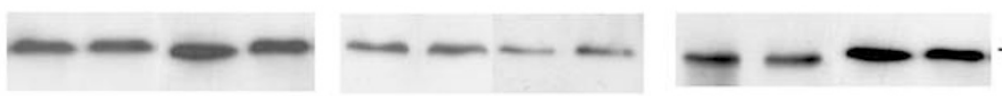

$-60 \mathrm{kDa}$

b PMAPK
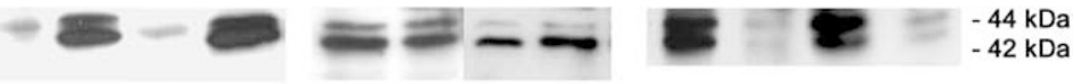

MAPK 1 MAPK 2


C $\mathrm{Bcl}-2$

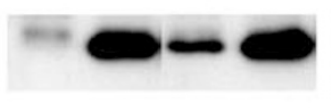

veh epo veh epo
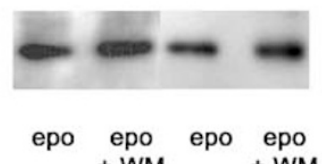

$$
+ \text { WM }
$$

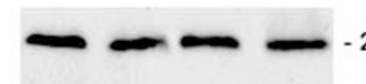

$-23 \mathrm{kDa}$

d

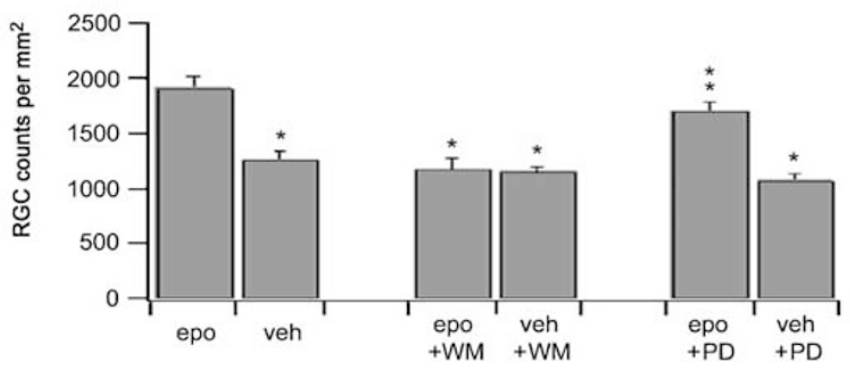

Figure 3 Intracellular signal transduction cascades modified by Epo treatment. In each column, different Western blots of the same retinal protein lysate are shown. (a) Western blot analysis of phosphorylated (pAkt) and unphosphorylated (Akt) Akt protein. Retinal protein lysates of animals treated with Epo or vehicle as well as under simultaneous treatment of Epo or vehicle together with WM (epo + WM; veh + WM) and following a combined treatment with PD 98059 (epo + PD; veh + PD) were analyzed. (b) Western blot analysis of phosphorylated (pMAPK 1 and 2) and unphosphorylated forms of MAPK 1 and 2. (c) Western blot analysis of Bcl-2 protein levels. (d) RGC counts at day 1 of MOG-EAE following Epo (epo) or vehicle (veh) treatment are compared with a combined treatment of Epo or vehicle and WM (epo + WM, veh + WM), or Epo or vehicle and PD 98059 (epo + PD, veh + PD). *Statistically significant when compared with the Epo monotherapy group. ${ }^{*}$ Statistically significant when compared with Epo monotherapy or combined treatment of vehicle and PD 98059 (one-way ANOVA followed by Duncan's test; $P<0.05$ ) 

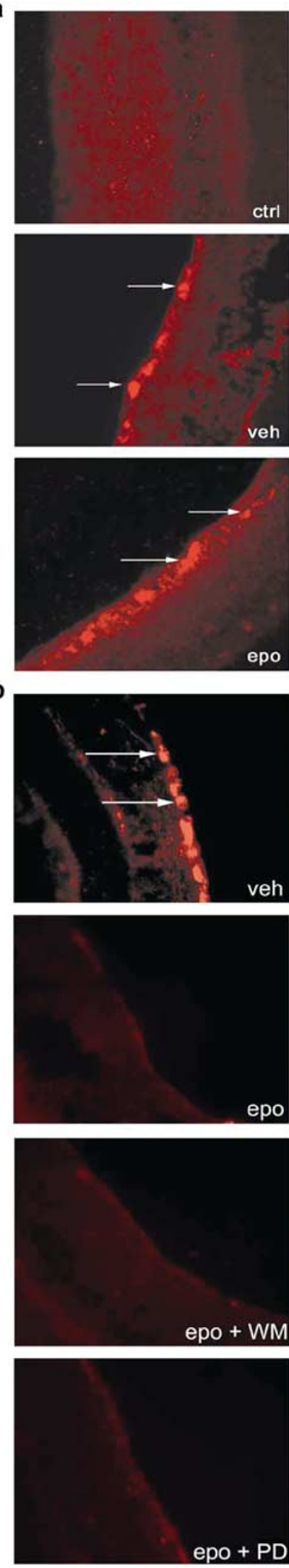

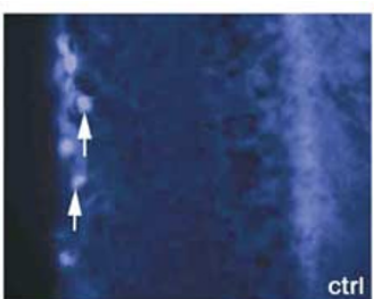

ctrl
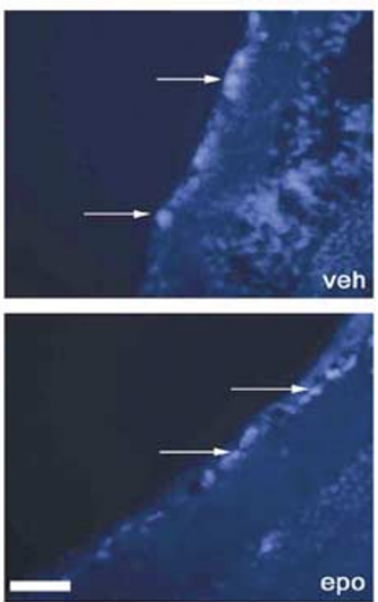

epo
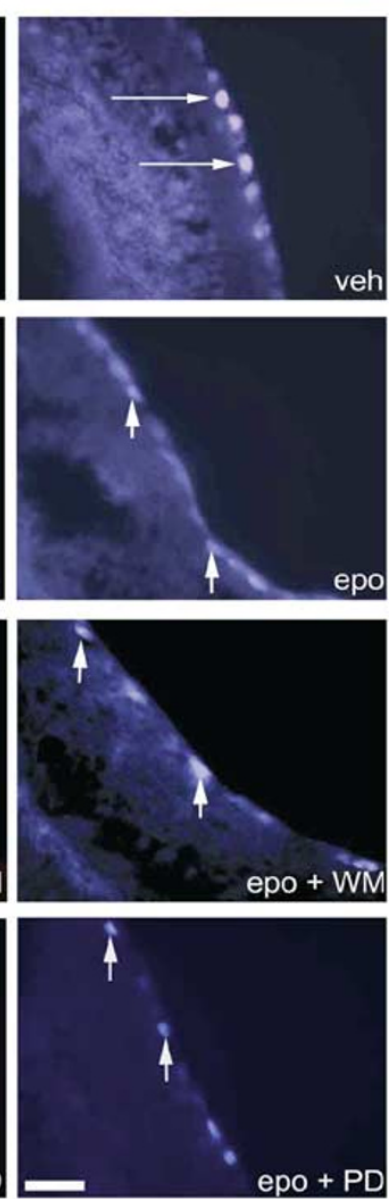

Inhibition of the phosphorylation of Akt but not of MAPKs abolishes the neuroprotective effect of Epo

To determine the functional relevance of the Epo-induced phosphorylation of the neuroprotective protein Akt, we treated rats with a combined therapy of Epo and wortmannin (WM), a specific inhibitor of PI3-K. WM was administered by intravitreal injections and its efficiency was proven by Western blot analysis (Figure 3a). After axotomy of the ON, the same treatment protocol of WM was shown to sufficiently suppress the neuroprotective effects of insulin-like growth factor-I or tumor necrosis factor- $\alpha .{ }^{27,28}$ Under this combined therapy of Epo and WM, RGC counts at day 1 of MOG-EAE were significantly decreased to $1177 \pm 91$ RGCs per square millimeter $(n=6)$ compared to $1917 \pm 99$ cells in the animal group that received Epo as a monotherapy $(n=8 ; P<0.05$; Figure 3d). WM itself $(n=6)$ had no noteworthy effect on RGC counts when compared to vehicle-treated animals $(1162 \pm 34$ versus $1262 \pm 74$ RGCs per square millimeter; Figure $3 d$ ). These data indicate that the activity of $\mathrm{PI} 3-\mathrm{K}$ is functionally relevant for the neuroprotective action of Epo in MOG-EAE. In contrast, the activity of endogenous PI3-K in MOG-EAE does not have any neuroprotective effect as treatment with WM did not decrease RGC counts notably below control levels.

Following applications of Epo together with intravitreal injections of WM, we observed an increased number of TUNEL-positive RGCs compared with Epo monotherapy. After this combined treatment, the number of TUNEL-positive RGCs per retinal section did not differ significantly from vehicle-treated controls (data not shown). In addition, at day 7 after immunization as well as at day 1 of MOG-EAE, the visual acuity determined by ERGs under this combined therapy $(0.1 \pm 0.07$ cycles per degree at day 7 p.i.; $0.07 \pm 0.04$ at day 1 of MOG-EAE; $n=8$ for each group) was similar to that of vehicle-treated controls, indicating that the improved RGC function was also abolished by application of the PI3-K inhibitor.

In addition, we analyzed whether the phosphorylation of MAPK 1 and 2 is functionally relevant in our animal model. Therefore, we used a combined treatment of systemic applications of Epo or vehicle, together with intravitreal injections of a specific inhibitor of MEK, PD 98059. In a previous study, treatment with this inhibitor was shown to suppress $R G C$ rescue due to monosialoganglioside treatment after axotomy of the ON. ${ }^{29}$ The efficiency of PD 98059 to inhibit phosphorylation of MAPK 1 and 2 was again proven by

Figure 4 Detection of EpoR and Bax in retinal sections. (a) Immunohistochemistry for EpoR expression in the retina. Double staining of representative retinal sections from a healthy control (ctrl), a vehicle-treated animal (veh), and an Epo-treated rat (epo) at day 1 of MOG-EAE. Specific staining for EpoR protein (red) is demonstrated in RGCs identified by FG-labeling (blue). Horizontal arrows indicate FG-labeled RGCs that show EpoR expression, whereas EpoR-negative RGCs are indicated by vertical arrows. Scale bar: $40 \mu \mathrm{m}$. (b) Immunohistochemistry for Bax protein expression (red) in the retina at day 1 of MOG-EAE in animals treated with vehicle (veh) or Epo (epo) monotherapy as well as following a combined treatment of Epo together with WM (epo + WM) or PD 98059 (epo + PD). RGCs were identified by FG-labeling (blue). Horizontal arrows indicate $F G$ labeled RGCs positive for Bax immunostaining. Vertical arrows signify FG labeled RGCs with no Bax expression. Scale bar: $40 \mu \mathrm{m}$ 
Western blot analysis (Figure 3b). These experiments revealed that the influence of MEK on the survival of RGCs under Epo injections is much smaller than that of PI3-K. RGC counts, following a combined treatment of Epo together with PD 98059 (1709.0 \pm 79 per square millimeter; $n=6$; Figure $3 d$ ), were still significantly higher than under vehicle therapy $(1262 \pm 74 ; n=8)$. PD 98059 treatment itself did not change RGC counts considerably when compared to vehicle therapy (1079.1 \pm 57 following treatment with PD 98059 versus $1262 \pm 74$ under vehicle therapy; $n=6$ versus 8 ; Figure 3d).

\section{Epo-induced regulation of $\mathrm{Bcl}-2$ and $\mathrm{Bax}$ does not depend on phosphorylation of Akt or MAPKs}

In order to investigate the Epo-dependent signal transduction in our MOG-EAE model further, we looked for a possible link between the $\mathrm{Bcl}-2$ family and $\mathrm{PI}-\mathrm{K}$. It has been demonstrated in a previous in vitro study in primary hippocampal neurons that inhibition of PI3-K blocked the antiapoptotic effects of insulin-like growth factor-I by decreasing Bcl-2 levels and increasing Bax expression. ${ }^{30}$ We performed Western blot analysis of $\mathrm{Bcl}-2$ in animals treated with Epo together with WM. In these animals, Bcl-2 levels were similar when compared to the ones of rats that were treated with daily injections of Epo as a monotherapy (Figure 3c). These data indicate that the Epo-induced upregulation of $\mathrm{Bcl}-2$ is not dependent on the activity of PI3-K. Additionally, we performed immunohistochemistry for Bax in animals treated with a combination of Epo and WM and compared the expression to the one in animals that had only received Epo. The expression of this proapoptotic member of the Bcl-2 family was unaffected by WM injections as well (Figure $4 b$ ).

In different cancer cell lines, inhibition of MEK activity was shown to correlate with a downregulation of expression levels of Bcl-2 and other members of the Bcl-2 family. ${ }^{31,32}$ To analyze whether in MOG-EAE under Epo treatment the activity of MEK determines the expression of $\mathrm{Bcl}-2$ or Bax, we performed Western blot analysis of $\mathrm{Bcl}-2$ as well as Bax immunostaining in animals treated with PD 98059. These experiments revealed that Bcl-2 and Bax levels under Epo application were unaffected by inhibition of MAPK phosphorylation (Figures $3 c$ and $4 b$ ).

\section{Discussion}

We demonstrate in an animal model of MS that Epo is an effective therapy that targets the neurodegenerative aspect of this neuro-inflammatory disease. In rats suffering from severe optic neuritis, Epo treatment significantly improved survival and function of RGCs, the neurons that form the axons of the ON. For the first time, we show in vivo that the antiapoptotic effects of Epo on these cells were mediated by an Epoinduced modification of the expression of Bcl-2 and Bax, as well as by activation of an intracellular neuroprotective pathway involving PI-3K/Akt. On the contrary, the detectable simultaneous activation of MAPKs under Epo treatment had only a small impact on RGC survival as revealed by experiments with application of a MEK inhibitor.
We have shown previously that MOG-induced EAE in rats especially reflects the neurodegenerative aspects of MS and, therefore, represents a suitable model for investigating potential neuroprotective therapies. ${ }^{10}$ Common therapeutic approaches of MS mainly focus on the inflammatory pathogenesis of this disease. ${ }^{33}$ Treating inflammation in autoimmune diseases does not automatically result in secondary neuroprotection, as we have demonstrated very recently in our MOG-EAE model. In contrast, degeneration of RGCs following severe optic neuritis was even promoted by high-dose methylprednisolone treatment. ${ }^{11}$ So far, no therapy directly targeting neuronal survival has been established for MS patients. MRI studies and analysis of brain biopsies confirmed that significant axonal and neuronal injury was observed even in early stages of this disease. ${ }^{34,35}$ Correspondingly, degeneration of neurons and axons in our MOG-EAE model occurred before disease onset. ${ }^{10}$

Therapeutic neuroprotective approaches involving the use of classical neurotrophic factors such as brain-derived neurotrophic factor or ciliary neurotrophic factor are difficult due to limitations in delivering these substances to their target cells. $^{36,37}$ The advantage of the 'atypical' neurotrophin Epo lies in its application mode and the good clinical tolerability. Epo is established as a long-term therapy to treat anemia in hemodialysis patients. In these patients, even over a period of more than 2 years, Epo applications were shown to increase erythropoiesis, ${ }^{38}$ indicating that this drug is not being inactivated by immunogenic reactions during long-term treatment. In a recent study of acute ischemic stroke in humans, intravenous treatment with Epo was well tolerated and an improvement in clinical outcome as well as a reduction in infarct size were observed. ${ }^{39}$ A different potentially neuroprotective approach would be targeting caspases. The use of caspase inhibitors as an antiapoptotic treatment strategy to protect neurons from premature cell death during chronic inflammatory autoimmune CNS diseases has been discussed in context with an in vitro study on neuronal cultures. ${ }^{40}$ However, caspase activation during the process of neuronal apoptosis is generally supposed to occur late in apoptotic cell death. ${ }^{41}$ Inhibition of a single execution system located downstream of the mitochondrium might not be sufficient to protect neurons which have already been damaged on proximal levels of their cell functions. Additionally, the antiapoptotic effect of caspase-3 inhibition has been shown to be transient and resulted in a delay rather than a permanent cell rescue of RGCs after axotomy of the ON. ${ }^{42}$

Epo acts as the main regulator of proliferation and differentiation of erythroid progenitor cells in the bone marrow. ${ }^{13}$ Neuroprotective effects of systemically administered Epo have been shown in animal models of focal cerebral ischemia, traumatic brain injury, subarachnoid hemorrhage, and spinal cord injury, ${ }^{19,43-45}$ but the relevance of the underlying molecular mechanisms in vivo remained still unclear. Additionally, Epo treatment rescued RGCs from apoptosis in a retinal ischemia model and improved RGC function determined by ERG recordings. ${ }^{46}$ Recently, we reported a loss of $65 \%$ of RGCs and a severe impairment of visual function occurring in early stages of MOG-EAE. ${ }^{22}$ In our 
present study, Epo treatment promoted the survival of about $50 \%$ of these neurons that underwent apoptosis in vehicletreated controls in our neuro-inflammatory animal model. Moreover, an improvement in pattern ERG recordings at day 7 after immunization as well as at day 1 of MOG-EAE was detectable in Epo-treated animals, indicating that those RGCs rescued from apoptotic neuronal cell death had preserved their electrophysiological functions. In agreement with a previous study that analyzed the effects of Epo treatment on MBP-EAE in Lewis rats, ${ }^{19}$ we observed a tendency towards a better clinical outcome in the Epo-treated animal group.

In a model of MBP-induced EAE, it has been shown that Epo reduced production of proinflammatory cytokines such as tumor necrosis factor- $\alpha$ and interleukin- 6 as well as proliferation and activation of microglia. ${ }^{20}$ In contrast to these findings, our histopathological analysis of the ONs revealed no differences in inflammatory infiltrates or demyelination in Epo-treated animals when compared with controls. This might be explained by the fact that MBP-EAE pathogenetically differs from the MOG-induced variant as it mainly represents the autoimmune T-cell response. ${ }^{9,47} \mathrm{We}$ are aware that we cannot fully exclude changes in subtypes or activation patterns of immune cells in our model. On the other hand, the results from our histopathological evaluation correlate well with the VEP recordings in which we observed no improvement in ON function following Epo treatment. From these observations, we conclude that systemically administered Epo does not influence or prevent the autoimmune reaction leading to disease manifestation in MOG-EAE, but exerts direct effects on RGCs. Antiapoptotic effects of Epo on immunopurified RGCs have been demonstrated recently in vitro, indicating that Epo acts directly on these neurons. ${ }^{48}$ Additionally, in this study intravitreal applications of Epo were shown to prevent RGC death after surgical transection of the $\mathrm{ON}$. As the intravitreal approach causes structural damage to the rat eye after repetitive applications, we used systemic applications of Epo in our present study. In our MOG-EAE model, two conditions are required for a direct action of Epo on RGCs: substantial amounts of Epo must reach the retina and EpoR should be present on RGCs in a sufficient amount. We demonstrated by ELISA that retinal Epo levels were substantially elevated following intraperitoneal injection of $5000 \mathrm{U}$ Epo per kilogram bodyweight. In particular, in neuro-inflammatory disorders, disruption of the BBB might promote the local enrichment and availability of Epo for neuronal cell types. In vehicle-treated rats, we also detected certain amounts of endogenous Epo by ELISA. Additionally, we demonstrated a considerable upregulation of EpoR on RGCs during MOGEAE. In agreement with the results of a previous study, ${ }^{46}$ no significant amount of EpoR protein was detectable on RGCs of healthy rats. We conclude that the upregulation of EpoR in combination with the endogenous production of Epo during MOG-EAE is part of a rescue mechanism to protect RGCs from apoptotic neuronal cell death.

Analogous to the well-known antiapoptotic action that Epo exerts on erythroid progenitor cells, ${ }^{15}$ neuroprotective effects of Epo can involve the upregulation of antiapoptotic members of the Bcl-2 family, as has been demonstrated in cerebral ischemia in rodents. ${ }^{49}$ In our recent study, we showed that during the development of MOG-EAE the Bcl-2 family is involved in RGC degeneration. ${ }^{10}$ An increase in the proapoptotic protein Bax occurred within RGCs, whereas the expression of Bcl-2 was reduced. Following Epo applications, we now observed a modulation of the expression levels of these two members of the Bcl-2 family, Bcl-2 and Bax, towards the antiapoptotic side, indicating that this cytokine can prevent at least some of the proapoptotic processes taking place in our rat EAE model.

Another neuroprotective pathway that was upregulated under Epo treatment in our MOG-EAE model involves PI3-K/ Akt. This is the second intracellular signal transduction cascade that we identified to be downregulated simultaneously with RGC degeneration during development of MOGEAE. ${ }^{10}$ Increased phosphorylation of $p$-Akt was also shown to be involved in neuroprotection due to Epo treatment in cultured cortical neurons under hypoxic conditions. ${ }^{43}$ Our present experiments with combined treatment of Epo together with WM, the naturally occurring inhibitor of $\mathrm{PI} 3-\mathrm{K},{ }^{50}$ revealed the functional relevance of this pathway as it completely abolished the Epo-induced RGC rescue. In our previous studies, p-Akt was demonstrated to play an important role in other neuroprotective treatments as the antiapoptotic effects of insulin-like growth factor-I and tumor necrosis factor- $\alpha$ after surgical transection of the ON were PI3-K dependent and could be abolished by application of WM. ${ }^{27,28}$ In our present in vivo study, the regulation of $\mathrm{Bcl}-2$ and $\mathrm{Bax}$ was independent from the activity of PI3-K as intravitreal injections of WM did not alter the expression levels of these proteins.

Another major signaling cascade which is activated by many cytokines is the well-described Ras/MAPK pathway. By Western blot analysis, we observed an increase of p-MAPK 1 and 2 in animals that had received daily injections of Epo. In previous studies on hematopoietic progenitors as well as in a study on neuronal cell cultures, an Epo-induced activation of the MEK/MAPK pathway has been demonstrated. ${ }^{43,51-53}$ To investigate the functional significance of this part of the Epoinduced signal transduction in vivo, we treated rats with a combination of Epo and PD 98059, a selective and cellpermeable inhibitor of the single upstream kinase MEK. ${ }^{54}$ Under this combined treatment, RGC counts were only moderately decreased when compared to Epo monotherapy, indicating that phosphorylation of MAPKs has a smaller impact on Epo-induced RGC survival than activation of PI3-K/ Akt. But we demonstrated recently that in MOG-EAE the proapoptotic effects of methylprednisolone on RGCs are mediated by a downregulation of $p-M A P K 2$. As Epo targets the same signal transduction cascade, a combined treatment of Epo together with methylprednisolone might prevent the increase of neuronal degeneration provoked by this established anti-inflammatory therapy.

In summary, our results demonstrate that Epo can serve as an effective neuroprotective treatment in a rat model of MS that reflects the neurodegenerative aspect of this disease in particular. Future combination therapies of Epo together with anti-inflammatory or immunomodulatory drugs might prevent or delay the development of a permanent neurological deficit and consecutive chronic disability in MS patients by reducing ongoing neuronal cell death. 


\section{Materials and Methods}

\section{Rats}

Female BN rats 8-10 weeks of age were used in all experiments. They were obtained from Charles River (Sulzfeld, Germany) and kept under environmentally controlled conditions without the presence of pathogens. These experiments have been approved by the local authorities in Braunschweig, Germany.

\section{Induction and evaluation of EAE}

Recombinant rat MOG $^{\text {lgd }}$ (rrMOG ${ }^{\text {Igd }}$ ), corresponding to the N-terminal sequence of rat MOG (amino acids 1-125), was expressed in Escherichia coli and purified to homogeneity as described previously. ${ }^{55}$ The rats were injected intradermally at the base of the tail with a total volume of $200 \mu \mathrm{l}$ inoculum, containing $50 \mu \mathrm{g} \mathrm{rrMOG}{ }^{\text {lgd }}$ in saline emulsified $(1: 1)$ with CFA (Sigma, St Louis, USA) containing $200 \mu \mathrm{g}$ heat-inactivated Mycobacterium tuberculosis (strain H 37 RA from Difco Laboratories, Detroit, USA). ${ }^{22}$ Rats were scored daily for clinical signs of EAE: grade 1, tail weakness or tail paralysis; grade 2, hindleg paraparesis or hemiparesis; grade 3 , hindleg paralysis or hemiparalysis; grade 4, complete paralysis (tetraplegy), moribund state, or death. Statistical significance of the manifestation of the disease and the clinical score at day 8 of MOG-EAE were assessed using Student's t-test.

\section{Systemic treatment of rats}

Animals were treated with daily i.p. injections of 2000,5000 , or $10000 \mathrm{U}$ recombinant human (r-hu) Epo (Janssen Cilag, Neuss, Germany) per kilogram bodyweight in $1 \mathrm{ml}$ of $0.9 \% \mathrm{NaCl}$ or vehicle from the day of immunization onward. The corresponding Epo- and vehicle-treated animal groups ( $n=6$ for each group) were killed at days 1 or 8 after manifestation of clinical symptoms.

\section{Intravitreal drug administration}

For intraocular injection of wortmannin (WM, $0.1 \mathrm{mM}$, dissolved in $15 \%$ dimethylsulfoxide; Sigma, St Louis, USA) or PD 98059 (2'-amino-3'. methoxyflavone, $20 \mathrm{mM}$ solution; Calbiochem, San Diego, USA), animals were anesthetized with diethylether. By means of a glass microelectrode, $2 \mu \mathrm{l}$ of each substance was injected into the vitreous space of each eye, puncturing the eye at the cornea-sclera junction. The injections were performed at days $0,4,7$, and 10 after immunization. The animals were killed on the day of manifestation of EAE.

\section{Retrograde labeling of RGCs}

Two weeks before immunization, RGCs were retrogradely labeled with FG. The BN rats were anesthetized by i.p. injection of Ketamin $10 \%$ $(0.65 \mathrm{ml} / \mathrm{kg}$; Atarost GmbH \& Co., Twistringen, Germany) together with Xylazin $2 \%(0.35 \mathrm{ml} / \mathrm{kg}$; Albrecht, Aulendorf, Germany) and placed in a stereotaxic frame. The skin was incised mediosagitally, and holes were drilled into the skull above each superior colliculus $(6.8 \mathrm{~mm}$ dorsal and $2 \mathrm{~mm}$ lateral from bregma). We injected stereotactically $2 \mu \mathrm{l}$ of the fluorescent dye FG ( $5 \%$ in normal saline; Fluorochrome Inc., Englewood, $\mathrm{CO}, \mathrm{USA}$ ) into both superior colliculi.

\section{Electrophysiological recordings}

The rats were anesthetized by i.p. injection of Ketamin $10 \%(0.65 \mathrm{ml} / \mathrm{kg}$; Atarost $\mathrm{GmbH} \&$ Co., Twistringen, Germany) together with Xylazin
$2 \% \quad(0.35 \mathrm{ml} / \mathrm{kg} ;$ Albrecht, Aulendorf, Germany) and mounted on a stereotaxic device. Recordings of VEPs and ERGs were performed as described previously. ${ }^{22}$ Visual stimuli were presented on a 17-in monitor (Acer View 76i) positioned $20 \mathrm{~cm}$ in front of the eye. Light flashes of $20 \mu \mathrm{s}$ duration were used at a rate of $1 \mathrm{~Hz}$, and bar stimulation consisted of vertical gratings of variable spatial frequency, alternating in phase with a temporal frequency of $1.8 \mathrm{~Hz}$ at $66 \%$ Michelson contrast (constant mean luminance $15 \mathrm{~cd} / \mathrm{m}^{2}$ ). Signals were amplified 10000 fold, bandpass filtered between 0.1 and $100 \mathrm{~Hz}$, and 128 events were averaged to improve the signal-to-noise ratio. Amplitudes of pattern ERG and pattern VEP as well as visual acuity values were determined as described previously. ${ }^{22}$

The electrophysiological recordings were performed in an Epo (5000 U/ $\mathrm{kg}$ ) and a vehicle-treated animal group, as well as in a group of rats that received Epo together with intravitreal injections of WM ( $n=8$ eyes for each group) at day 7 after immunization as well as at day 1 of MOG-EAE. In each case, ERG and VEP potentials in response to flash or pattern stimulation of $3,6,12,24,36$, and 48 alternating bars were analyzed. Immediately after the end of the second recording session, animals received an overdose of chloralhydrate. Retinas were removed for RGC counting and ONs were taken for histopathological evaluation. Statistical significance of visual acuities was assessed using one-way ANOVA followed by the Duncan's test.

\section{Quantification of RGC density}

Rats received an overdose of chloralhydrate and were perfused via the aorta with $4 \%$ paraformaldehyde (PFA) in PBS at days 1 and 8 of EAE. Retinas were dissected, flat-mounted on glass slides and examined by fluorescence microscopy (Axioplan 2, Zeiss, Göttingen, Germany) using a DAPI filter ( $315 / 395 \mathrm{~nm})$. RGC densities were determined as described. ${ }^{22}$ Cell counts were performed by two independent investigators following a blinded protocol. Data are given as mean \pm s.e.m. Statistical significance was assessed using one-way ANOVA followed by the Duncan's test.

\section{Immunohistochemistry}

Immunohistochemistry was performed on transverse cryostat microtome sections ( $18 \mu \mathrm{m}$ thick) of retinas of day 1 of EAE. DNA fragmentation of cells undergoing apoptosis was analyzed by the TUNEL (terminal deoxynucleotidyl transferase-mediated biotinylated UTP nick end labeling) method. Sections were incubated with $50 \mathrm{U}$ terminal transferase and $1 \mathrm{mM}$ biotin-dUTP in the presence of $1.5 \mathrm{mM} \mathrm{CoCl}_{2}, 0.2 \mathrm{M} \mathrm{K}^{+}$-cacodylate, and $25 \mathrm{mg} / \mathrm{ml} \mathrm{BSA}$ for $90 \mathrm{~min}$ at $37^{\circ} \mathrm{C}$. Incorporated biotinylated nucleotides were detected by incubation with fluorescein isothiocyanate-conjugated streptavidin and examined by fluorescence microscopy. Rabbit polyclonal antibodies were used to localize activated caspase-3 (1:1000; CM1; PharMingen International, San Diego, USA), Bax (1:200; sc-526; Santa Cruz Biotechnology, Inc., CA, USA), and EpoR (1: 1000; sc-697; Santa Cruz Biotechnology, Inc., CA, USA). A rabbit polyclonal antibody IgG-Cy3 $(1: 1000)$ was used as secondary antibody. Sections were preincubated with $10 \%$ normal goat serum (NGS) ( $1 \mathrm{~h}$, room temperature (RT)), followed by the primary antibody (overnight, $4^{\circ} \mathrm{C}$ ) and Cy-3-conjugated goat antirabbit IgG in 10\% NGS (1 h, RT; Dianova, Hamburg, Germany). The number of TUNEL or active caspase-3-positive RGCs per retinal section was determined by two independent investigators following a blinded protocol. Eight retinal sections comparable in size and location were used. Statistical significance was assessed using Student's $t$-test. 


\section{Western blotting}

For Western blot analysis, animals received an overdose of chloralhydrate $6 \mathrm{~h}$ after the last systemic application of Epo and $4 \mathrm{~h}$ after the last intravitreal injection of WM or PD 98059. The eyes were removed, and the retinas dissected. The retinas were homogenized and lysed $(150 \mathrm{mM}$ $\mathrm{NaCl}, 50 \mathrm{mM}$ Tris, pH 8.0, $2 \mathrm{mM}$ EDTA, and 1\% Triton, containing $0.1 \mathrm{mM}$ PMSF and $2 \mu \mathrm{g} / \mathrm{ml}$ pepstatin, leupeptin, and aprotinin) for $20 \mathrm{~min}$ on ice. Cell debris were then pelleted at $13000 \times g$ for $15 \mathrm{~min}$. The protein concentration of the supernatant was determined using the BCA reagent (Pierce, Rockford, IL, USA). After separation by reducing SDS-PAGE of the lysates ( $20 \mu \mathrm{g}$ protein per lane), proteins were transferred to a polyvinylidene difluoride membrane and blocked with $5 \%$ skim milk in $0.1 \%$ Tween 20 in PBS-T. The membranes were incubated with the primary antibody against phospho-Akt (9271, New England Biolabs GmbH, Schwalbach, Germany; $1: 1000$ in 1\% skim milk in PBS-T), Akt (9272, New England Biolabs GmbH, Schwalbach, Germany; 1: 1000 in 5\% skim milk), phospho-MAPK 1 and 2 (9106, New England Biolabs $\mathrm{GmbH}$, Schwalbach, Germany; $1: 200$ in 1\% skim milk in PBS-T), or Epo (sc-7956, Santa Cruz Biotechnology, Inc., CA, USA; 1:500 in 5\% skim milk). Membranes were washed in PBS-T and then incubated with HRPconjugated secondary antibodies against rabbit IgG (Santa Cruz Biotechnology, Inc., CA, USA; 1:2500 in 1\% skim milk). For Western blot analysis of Bcl-2 levels (sc-7382, Santa Cruz Biotechnology, Inc., CA, USA; $1: 200 ; 5 \%$ skim milk), an HRP-conjugated secondary antibody against mouse IgG was used (Santa Cruz, Biotechnology, Inc., CA, USA; $1: 2000$ in 1\% skim milk in PBS-T). MAPK 1 and 2 protein levels were detected using a primary antibody (sc-93-G, Santa Cruz Biotechnology Inc., CA, USA) diluted 1:500 in 1\% skim milk in PBS-T, and an HRPconjugated secondary antibody against goat IgG (Santa Cruz Biotechnology Inc., CA, USA; 1 : 10000 in PBS-T). Labeled proteins were detected using the ECL-plus reagent (Amersham, Arlington Heights, IL, USA). To estimate the relative expression levels of the different proteins, the expression patterns were analyzed in the same retinal protein lysate. At least four different retinal protein lysates were used to study each effect.

\section{ELISA}

In order to determine retinal Epo levels by ELISA, retinal supernatants of vehicle-treated rats were investigated and compared with those of Epotreated animals (intraperitoneal application of $5000 \mathrm{U} / \mathrm{kg}$ daily) at day 15 after immunization with MOG. To achieve sufficient amounts of material for ELISA, retinas of six animals treated with vehicle or Epo were pooled. To obtain retinal supernatants for ELISA testing, the eyes were removed $1 \mathrm{~h}$ after the last application of Epo or vehicle. Retinas were dissected and homogenized on ice in $200 \mu$ PBS. Cell debris were pelleted at $13000 \times g$ for $15 \mathrm{~min}$. Retinal Epo levels were detected using the quantitative sandwich enzyme immunoassay technique. A commercially available ELISA kit (R\&D Systems, Minneapolis, USA) was used and the provider's instructions were followed. To obtain a standard curve, the $E$. coliexpressed Quantikine M rat kit standard was used. Epo levels were normalized to the protein concentration of the supernatants determined by using the BCA reagent (Pierce, Rockford, IL, USA).

\section{Hematocrit}

Hematocrit levels were determined in blood samples from three different animal groups, containing four animals each: healthy controls, vehicletreated rats at day 8 of MOG-EAE, and rats that had received a daily treatment of $5000 \mathrm{U}$ Epo per kilogram administered intraperitoneally from the day of immunization onward until day 8 of MOG-EAE.

\section{Histopathology}

Histological evaluation was performed on $4 \%$ paraformaldehyde-fixed and paraffin-embedded sections of ONs. Slices $4 \mu \mathrm{m}$ thick were stained with hematoxylin and eosin, Luxol-fast blue, and Bielschowky's silver impregnation to assess inflammation, demyelination, and axonal pathology. Axonal densities were determined in vertical sections of the ONs stained with Bielschowsky's silver impregnation. Measurements were performed at 1000 -fold magnification using a 25 -point Zeiss eyepiece. ${ }^{56}$ The number of axons in each ON was counted in at least 14 standardized microscopic fields of $2500 \mu \mathrm{m}^{2}$. Mean axon density was calculated for each ON. The surface area of the ON was measured with the NIH-Image and the total number of axons per ON was extrapolated from the calculated mean axon density. Demyelinated areas were determined as percentage of the whole transverse ON cross section. The investigators who performed neuropathological examinations were blinded to the electrophysiological results.

\section{Acknowledgements}

This work was supported by the Gemeinnützige Hertie-Stiftung and Biogen Idec. MBS and the junior research group (RD, DM, CS) were funded by the medical faculty of the University of Göttingen. Erythropoietin was kindly provided by Janssen Cilag, Neuss, Germany. We thank Inna Boger and Birgit Kramer for expert technical assistance.

\section{References}

1. Ferguson B, Matyszak MK, Esiri MM and Perry VH (1997) Axonal damage in acute multiple sclerosis lesions. Brain 120: 393-399

2. Trapp BD, Peterson J, Ransohoff RM, Rudick R, Mork S and Bo L (1998) Axonal transection in the lesions of multiple sclerosis. N. Engl. J. Med. 338: 278-285

3. Davie CA, Barker GJ, Webb S, Tofts PS, Thompson AJ, Harding AE, McDonald WI and Miller DH (1995) Persistent functional deficit in multiple sclerosis and autosomal dominant cerebellar ataxia is associated with axon loss. Brain 118: 1583-1592

4. Lovas G, Szilagyi N, Majtenyi K, Palkovits M and Komoly S (2000) Axonal changes in chronic demyelinated cervical spinal cord plaques. Brain 123: 308-317

5. Trapp BD, Ransohoff R and Rudick R (1999) Axonal pathology in multiple sclerosis: relationship to neurologic disability. Curr. Opin. Neurol. 12: 295-302

6. Peterson JW, Bo L, Mark S, Chang A and Trapp BD (2001) Transected neurites, apoptotic neurons, and reduced inflammation in cortical multiple sclerosis lesions. Ann. Neurol. 50: 389-400

7. Storch MK, Stefferl A, Brehm U, Weissert R, Wallstrom E, Kerschensteiner M, Olsson T, Linington C and Lassmann $\mathrm{H}$ (1998) Autoimmunity to myelin oligodendrocyte glycoprotein in rats mimics the spectrum of multiple sclerosis pathology. Brain Pathol. 8: 681-694

8. Stefferl A, Brehm U, Storch MK, Lambracht-Washington D, Bourquin C, Wonigeit K, Lassmann H and Linington C (1999) Myelin oligodendrocyte glycoprotein induces experimental autoimmune encephalomyelitis in the 'resistant' Brown Norway rat: disease susceptibility is determined by MHC and MHC-linked effects on the B cell response. J. Immunol. 163: 40-49

9. Iglesias A, Bauer J, Litzenburger T, Schubart A and Linington C (2001) T- and $\mathrm{B}$-cell responses to myelin oligodendrocyte glycoprotein in experimental autoimmune encephalomyelitis and multiple sclerosis. Glia 36: 220-234 
10. Hobom M, Storch MK, Weissert R, Maier K, Radhakrishnan A, Kramer B, Bähr $M$ and Diem R (2004) Mechanisms and time course of neuronal degeneration in experimental autoimmune encephalomyelitis. Brain Pathol. 14: 148-157

11. Diem R, Hobom M, Maier K, Weissert R, Storch MK, Meyer R and Bähr M (2003) Methylprednisolone increases neuronal apoptosis during autoimmune CNS inflammation by inhibition of an endogenous neuroprotective pathway. J. Neurosci. 23: 6993-7000

12. Kornek B, Storch MK, Weissert R, Wallstroem E, Stefferl A, Olsson T, Linington C, Schmidbauer M and Lassmann H (2000) Multiple sclerosis and chronic autoimmune encephalomyelitis: a comparative quantitative study of axonal injury in active, inactive, and remyelinated lesions. Am. J. Pathol. 157: 267-276

13. Jelkmann W (1992) Erythropoietin: structure, control of production, and function. Physiol. Rev. 72: 449-489

14. Ihle JN and Kerr IM (1995) Jaks and Stats in signaling by the cytokine receptor superfamily. Trends Genet. 11: 69-74

15. Silva M, Grillot D, Benito A, Richard C, Nunez G and Fernandez-Luna JL (1996) Erhythropoietin can promote erythroid progenitor survival by repressing apoptosis through $\mathrm{Bcl}-\mathrm{XI}$ and $\mathrm{Bcl}-2$. Blood 88: 1576-1582

16. Konishi Y, Chui CH, Hirose H, Kunishita T and Tabira T (1993) Trophic effect of erythropoietin and other hemotopoietic factors on central cholinergic neurons in vitro and in vivo. Brain Res. 609: 29-35

17. Sadamoto Y, Igase K, Sakanaka M, Sato K, Otsuka H, Sakaki S, Masuda S and Sasaki R (1998) Erythropoietin prevents place navigation disability and cortical infarction in rats with permanent occlusion of the middle cerebral artery. Biochem. Biophys. Res. Commun. 253: 26-32

18. Bernaudin M, Marti HH, Roussel S, Divoux D, Nouvelot A, Mackenzie ET and Petit E (1999) A potential role for erythropoietin in focal permanent cerebra ischemia in mice. J. Cereb. Blood Flow Metab. 19: 643-651

19. Brines ML, Ghezzi P, Keenan S, Agnello D, de Lanerolle NC, Cerami $C$ and Cerami A (2000) Erythropoietin crosses the blood-brain barrier to protect against experimental brain injury. Proc. Natl. Acad. Sci. USA 97: 10526-10531

20. Agnello D, Bigini $P$, Villa $P$, Mennini $T$, Cerami $A$, Brines $M L$ and Ghezzi $P$ (2002) Erythropoietin exerts an anti-inflammatory effect on the CNS in a model of experimental autoimmune encephalomyelitis. Brain Res. 952: 128-134

21. Maffei $L$ and Fiorentini $A$ (1981) Electroretinographic responses to alternating gratings before and after section of the optic nerve. Science 211: 953-955

22. Meyer R, Weissert R, Diem R, Storch MK, de Graaf KL, Kramer B and Bähr M (2001) Acute neuronal apoptosis in a rat model of multiple sclerosis. J. Neurosci. 21: 6214-6220

23. Franke TF, Kaplan DR and Cantley LC (1997) PI3K: downstream AKT ion blocks apoptosis. Cell 88: 435-437

24. Seger $R$ and Krebs EG (1995) The MAPK signaling cascade. FASEB J. 9 : $726-735$

25. Merry DE and Korsmeyer SJ (1997) Bcl-2 gene family in the nervous system. Annu. Rev. Neurosci. 20: 245-267

26. Oltvai ZN, Milliman CL and Korsmeyer SJ (1993) Bcl-2 heterodimerizes in vivo with a conserved homolog, Bax, that accelerates programmed cell death. Cell 74: 609-619

27. Kermer $P$, Klöcker N, Labes $M$ and Bähr $M(2000)$ Insulin-like growth factor-I protects axotomized rat retinal ganglion cells from secondary death via PI3-Kdependent Akt phosphorylation and inhibition of caspase-3 in vivo. J. Neurosci. 20: 722-728

28. Diem R, Meyer R, Weishaupt JH and Bähr M (2001) Reduction of potassium currents and phosphatidylinositol 3-kinase-dependent Akt phosphorylation by tumor necrosis factor-alpha rescues axotomized retinal ganglion cells from retrograde cell death in vivo. J. Neurosci. 21: 2058-2066

29. Choi JS, Kim JA and Joo CK (2003) Activation of MAPK and CREB by GM1 induces survival of RGCs in the retina with axotomized nerve. Invest. Ophthalmol. Vis. Sci. 44: 1747-1752

30. Matsuzaki H, Tamatani M, Mitsuda N, Namikawa K, Kiyama H, Miyake S and Tohyama M (1999) Activation of Akt kinase inhibits apoptosis and changes in Bcl-2 and Bax expression induced by nitric oxide in primary hippocampal neurons. J. Neurochem. 73: 2037-2046

31. Boucher MJ, Morisset J, Vachon PH, Reed JC, Laine J and Rivard N (2000) MEK/ERK signaling pathway regulates the expression of $\mathrm{Bcl}-2, \mathrm{Bcl}-\mathrm{X}(\mathrm{L})$, and Mcl-1 and promotes survival of human pancreatic cancer cells. J. Cell Biochem. 79: 355-369

32. Zheng B, Fiumara P, Li Y, Georgakis GV, Snell V, Younes M, Vauthey JN, Carbone A and Younes A (2003) MEK/ERK pathway is aberrantly active in
Hodgkin disease: a shared signaling pathway among CD30, CD40, and RANK that regulates cell proliferation and survival. Blood 102: 1019-1027

33. Steinman $L$ (2001) Immunotherapy of multiple sclerosis: the end of the beginning. Curr. Opin. Immunol. 13: 597-600

34. Simon JH, Jacobs LD, Campion MK, Rudick RA, Cookfair DL, Herndon RM, Richert JR, Salazar AM, Fischer JS, Goodkin DE, Simonian N, Lajaunie M, Miller DE, Wende K, Martens-Davidson A, Kinkel RP, Munschauer III FR and Brownscheidle CM (1999) A longitudinal study of brain atrophy in relapsing multiple sclerosis. The Multiple Sclerosis Collaborative Research Group (MSCRG). Neurology 13: 139-148

35. Kuhlmann T, Lingfeld G, Bitsch A, Schuchardt J and Brück W (2002) Acute axonal damage in multiple sclerosis is most extensive in early disease stages and decreases over time. Brain 125: 2202-2212

36. Penn RD, Kroin JS, York MM and Cedarbaum JM (1997) Intrathecal ciliary neurotrophic factor delivery for treatment of amyotrophic lateral sclerosis (phase I trial). Neurosurgery 40: 94-99

37. Abicht A and Lochmuller H (1999) Technology evaluation: CRIB (CNTF delivery). CytoTherapeutics Inc. Curr. Opin. Mol. Ther. 1: 645-650

38. Horina JH, Schmid CR, Roob JM, Winkler HM, Samitz MA, Hammer HF, Pogglitsch $\mathrm{H}$ and Krejs GJ (1991) Bone marrow changes following treatment of renal anemia with erythropoietin. Kidney Int. 40: 917-922

39. Ehrenreich H, Hasselblatt M, Dembowski C, Cepek L, Lewczuk P, Stiefel M, Rustenbeck HH, Breiter N, Jacob S, Knerlich F, Bohn M, Poser W, Ruther E, Kochen M, Gefeller O, Gleiter C, Wessel TC, De Ryck M, Itri L, Prange H, Cerami A, Brines M and Siren AL (2002) Erythropoietin therapy for acute stroke is both safe and beneficial. Mol. Med. 8: 495-505

40. Cid C, Alvarez-Cermeno JC, Regidor I, Plaza J, Salinas M and Alcazar A (2003) Caspase inhibitors protect against neuronal apoptosis induced by cerebrospinal fluid from multiple sclerosis patients. J. Neuroimmunol. 136: $119-124$

41. Bähr M (2000) Live or let die - retinal ganglion cell death and survival during development and in the lesioned adult CNS. Trends Neurosci. 23: 483-490

42. Kermer P, Klöcker N and Bähr M (1999) Long-term effect of inhibition of ced 3like caspases on the survival of axotomized retinal ganglion cells in vivo. Exp. Neurol. 158: 202-205

43. Siren AL, Fratelli M, Brines M, Goemans C, Casagrande S, Lewczuk P, Keenan S, Gleiter C, Pasquali C, Capobianco A Mennini T, Heumann R, Cerami A, Ehrenreich $\mathrm{H}$ and Ghezzi P (2001) Erythropoietin prevents neuronal apoptosis after cerebral ischemia and metabolic stress. Proc. Natl. Acad. Sci. USA 98: 4044-4049

44. Buemi M, Grasso G, Corica F, Calapai G, Salpietro FM, Casuscelli T, Sfacteria A, Aloisi C, Alafaci C, Sturiale A, Frisina N and Tomasello $F(2000)$ In vivo evidence that erythropoietin has a neuroprotective effect during subarachnoid hemorrhage. Eur. J. Pharmacol. 392: 31-34

45. Gorio A, Gokmen N, Erbayraktar S, Yilmaz O, Madaschi L, Cichetti C, Di Giulio AM, Vardar E, Cerami A and Brines M (2002) Recombinant human erythropoietin counteracts secondary injury and markedly enhances neurological recovery from experimental spinal cord trauma. Proc. Natl. Acad. Sci. USA 99: 9450-9455

46. Junk AK, Mammis A, Savitz SI, Singh M, Roth S, Malhotra S, Rosenbaum PS, Cerami A, Brines M and Rosenbaum DM (2002) Erythropoietin administration protects retinal neurons from acute ischemia-reperfusion injury. Proc. Natl. Acad. Sci. USA 99: 10659-10664

47. Wekerle H, Kojima K, Lannes-Vieira J, Lassmann H and Linington C (1994) Animal models. Ann. Neurol. 36: S47-S53

48. Weishaupt JH, Rohde G, Pölking E, Siren AL, Ehrenreich H and Bähr M (2004) Erythropoietin protects against axotomy-induced apoptosis in rat retinal ganglion cells. Invest. Ophthalmol. Vis. Sci. 45: 1514-1522

49. Wen TC, Sadamoto Y, Tanaka J, Zhu PX, Nakata K, Ma YJ, Hata R and Sakanaka M (2002) Erythropoietin protects neurons against chemical hypoxia and cerebral ischemic injury by up-regulating Bcl-XI expression. J. Neurosci. Res. 67: 795-803

50. Alessi DR, Andjelkovic M, Caudwell B, Cron P, Morrice N, Cohen $P$ and Hemmings BA (1996) Mechanisms of activation of protein kinase B by insulin and IGF-1. EMBO J. 15: 6541-6551

51. Bittorf T, Jaster R and Brock J (1994) Rapid activation of the MAP kinase pathway in hematopoietic cells by erythropoietin, granulocyte-macrophage colony-stimulating factor and interleukin-3. Cell Signal 6: 305-311 
Neuroprotective effects of Epo in EAE MB Sättler (née Hobom) et al

52. Koshimura K, Murakami Y, Sohmiya M, Tanaka J and Kato Y (1999) Effects of erythropoietin on neuronal activity. J. Neurochem. 72: 2565-2572

53. Sui X, Krantz SB and Zhao ZJ (2000) Stem cell factor and erythropoietin inhibit apoptosis of human erythroid progenitor cells through different signalling pathways. Br. J. Haematol. 110: 63-70

54. Kültz D, Madhany S and Burg MB (1998) Hyperosmolality causes growth arrest of murine kidney cells. Induction of GADD45 and GADD153 by osmosensing via stress-activated protein kinase 2. J. Biol. Chem. 273: 13645-13651
55. Weissert $\mathrm{R}$, Wallstrom $\mathrm{E}$, Storch MK, Stefferl A, Lorentzen J Lassmann $\mathrm{H}$, Linington $\mathrm{C}$ and Olsson T (1998) MHC haplotypedependent regulation of MOG-induced $\mathrm{EAE}$ in rats. J. Clin. Invest. 102: 1265-1273

56. Brück W, Bitsch $A$, Kulenda $H$, Brück $Y$, Stiefel $M$ and Lassmann $H$ (1997) Inflammatory central nervous system demyelination: correlation of magnetic resonance imaging findings with lesion pathology. Ann. Neurol. 42: 783-793 